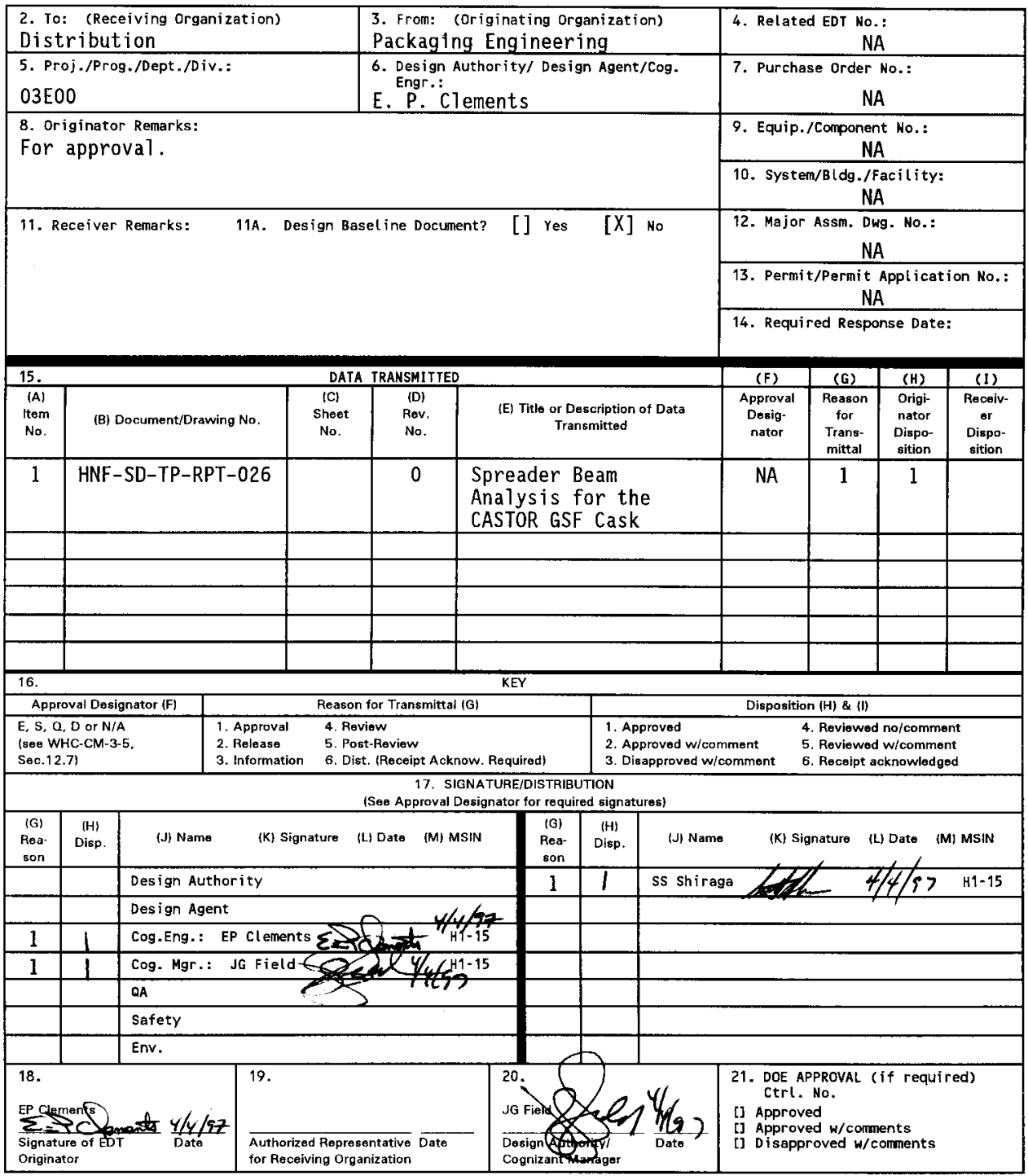

BD-7400-172-2 (05/96) GEF097 


\title{
Spreader Beam Analysis for the CASTOR GSF Cask
}

\author{
E. P. Clements
}

Rust Federal Services Inc. Northwest 0perations, Richland, WA 99352

U.S. Department of Energy Contract DE-AC06-96RL13200

$\begin{array}{lll}\text { EDT/ECN: } & \text { EDT } 619562 & \text { UC: } 512 \\ \text { Org Code: } & 03 E 00 & \text { Charge Code: POFK06 } \\ \text { B\&R Code: } 820201000 & \text { Total Pages: }-3433 \mathrm{gB}\end{array}$

Key Words: spreader beam, CASTOR GSF cask, load test

Abstract: The purpose of this report is to document the results of the $150 \%$ rated capacity load test that was performed by DynCorp Hoisting and Rigging for the CASTOR GSF special lifting beams.

TRADEMARK DISCLAIMER, Reference herein to any specific comercial product, process, or service by trade name, trademark, manufacturer, or otherwise, does not necessarily constitute or imply its endorsement, recommendation, or favoring by the United States Government or any agency thereof or its contractors or subcontractors.

Printed in the United States of America. To obtain copies of this document, contact: Document Control Services, P.O. Box 950, Mailstop H6-08, Richland WA 99352, Phone (509) 372-2420; Fax (509) 376-4989.
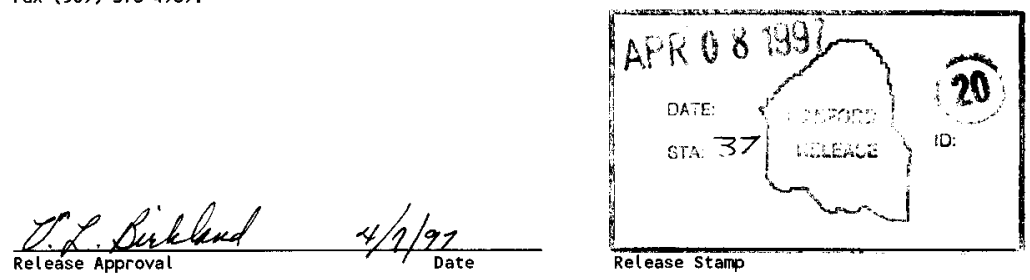

\section{Approved for Public Release}


CONTENTS

1.0 INTRODUCTION $\ldots \ldots \ldots \ldots \ldots \ldots \ldots \ldots \ldots \ldots \ldots \ldots \ldots \ldots \ldots$

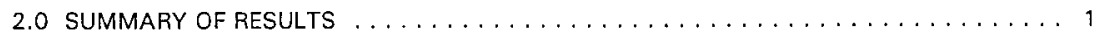

3.0 CONCLUSION $\ldots \ldots \ldots \ldots \ldots \ldots \ldots \ldots \ldots \ldots \ldots \ldots \ldots \ldots \ldots \ldots$

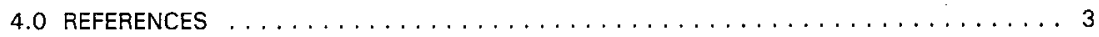

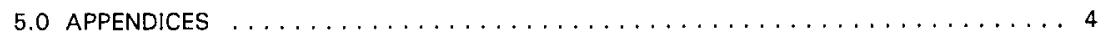

5.1 ORIGINAL GERMAN LOAD TESTS $\ldots \ldots \ldots \ldots \ldots \ldots \ldots \ldots \ldots \ldots \ldots$

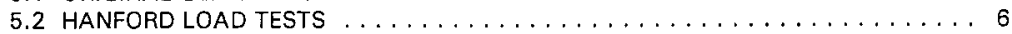

5.3 CASTOR LIFTING BEAM EVALUATION $\ldots \ldots \ldots \ldots \ldots \ldots \ldots \ldots \ldots$

\section{LIST OF FIGURES}

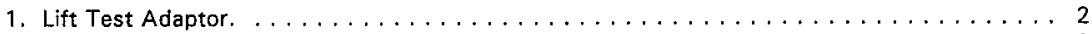

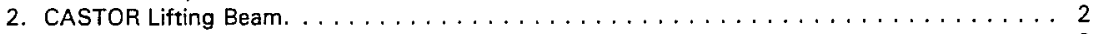

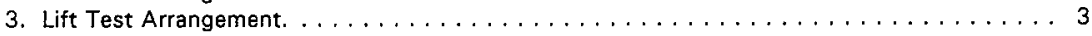

\section{LIST OF TERMS}

ANSI American National Standards Institute

ASTM American Society for Testing and Materials

IAEA International Atomic Energy Agency

SARP Safety Analysis Report for Packaging 
HNF-SD-TP-RPT-026 Rev. 0

\section{SPREADER BEAM ANALYSIS FOR THE CASTOR GSF CASK}

\subsection{INTRODUCTION}

The purpose of this report is to document the results of the $150 \%$ rated capacity load test performed by DynCorp Hoisting and Rigging on the CASTOR GSF special cask lifting beams. The two lifting beams were originally rated and tested at $20,000 \mathrm{~kg}(44,000 \mathrm{lb})$ by the cask manufacturer in Germany. The testing performed by DynCorp rated and tested the lifting beams to $30,000 \mathrm{~kg}(66,000 \mathrm{lb})+0 \%,-5 \%$, for Hanford Site use.

The CASTOR GSF cask, used to transport Isotopic Heat Sources (canisters), must be lifted with its own designed lifting beam system (Figures 1, 2, and 3). As designed, the beam material is RSt 37-2 (equivalent to American Society for Testing and Materials [ASTM] A-570), the eye plate is St 52-2 (equivalent to ASTM A-516), and the lifting pin is St 50 (equivalent to ASTM A-515). The beam has two opposing $58 \mathrm{~mm}$ (2.3 in.) diameter by $120 \mathrm{~mm}$ (4.7 in.) length, high grade steel pins that engage the cask for lifting. The pins have a manual locking mechanism to prevent disengagement from the casks. The static, gross weight (loaded) of the cask $18,640 \mathrm{~kg}$ $(41,000 \mathrm{lb})$ on the pins prevents movement of the pins during lifting. This is due to the frictional force of the cask on the pins when lifting begins.

\subsection{SUMMARY OF RESULTS}

The two lifting beams (1 $\mathrm{A}$ and $1 \mathrm{~B})$ delivered as auxiliary equipment with the CASTOR GSF casks were designed, built, tested, and used by the cask manufacturer in Germany (Section 5.1). The beams were built and tested to International Atomic Energy Agency (IAEA) requirements. For use at the Hanford Site, the beams shall meet the requirements listed in the Hanford Site Hoisting and Rigging Manual, Section 11.0, "Below-the-Hook-Lifting Devices" (RL 1993) and the American National Standard Institute (ANSI) N14.6, Radioactive Materials Special Lifting Devices for Shipping Containers Weighing $10,000 \mathrm{lb}(4500 \mathrm{~kg}$ ) or More (ANSI 1993). To meet the Hanford Site and ANSI criteria, both lifting beams were analyzed to ensure 3 to 1 against yield, requirements are met (Section 5.3). The beams were physically load tested (Section 5.2) in accordance with the Hanford Site Hoisting and Rigging Manual, Section 11.0, "Below-the-Hook-Lifting Devices" and ANSI N14.6, Section 6.3(a), "Testing," to verify continuing compliance. The two lifting beams were tested to $150 \% 30,000 \mathrm{~kg}(66,000 \mathrm{lb})+0 \%,-5 \%$ for an actual load test of $29,345 \mathrm{~kg}$ $(64,560 \mathrm{lb})$ held for 10 minutes. This test was performed with certified weights and rigging. The beams were inspected after testing as described in ANSI 6.3 (a) and no discrepancies were found.

\subsection{CONCLUSION}

The two CASTOR GSF cask lifting beams are only intended and designed for their particular use as described in the CASTOR Safety Analysis Report for Packaging (SARP)

(HNF-SD-TP-SARP-021). The beams meet both the Hanford Site Hoisting and Rigging Manual (Section 11.0) and ANSI N14.6 (Section 6.3.1[a]) criteria for use on the Hanford Site. Analysis demonstrates the beams meet a safety factor of 3 to 1 against yield for the approved load. The Hanford Site approved rating is $29,345 \mathrm{~kg}(64,500 \mathrm{lb})$. 
HNF-SD-TP-RPT-026 Rev. 0

Figure 1. Lift Test Adaptor.
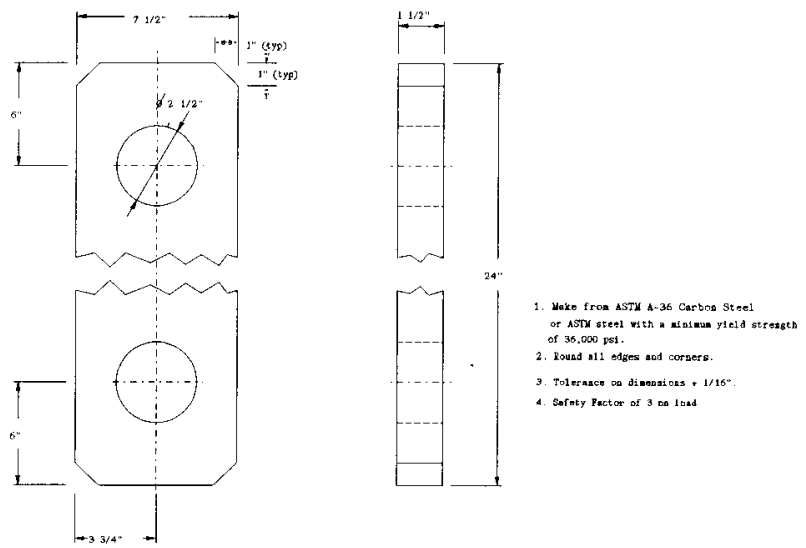

S. S. Shi rage 3/10/97

Figure 2. CASTOR Lifting Beam.

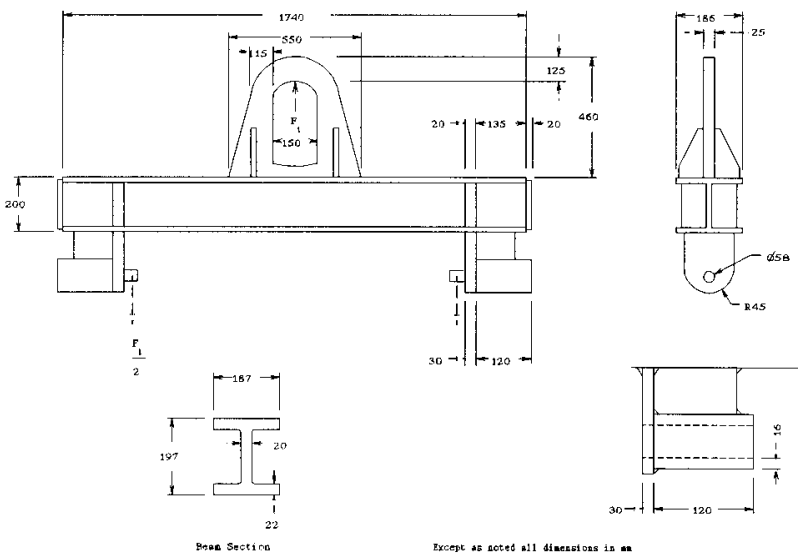


Figure 3. Lift Test Arrangement.

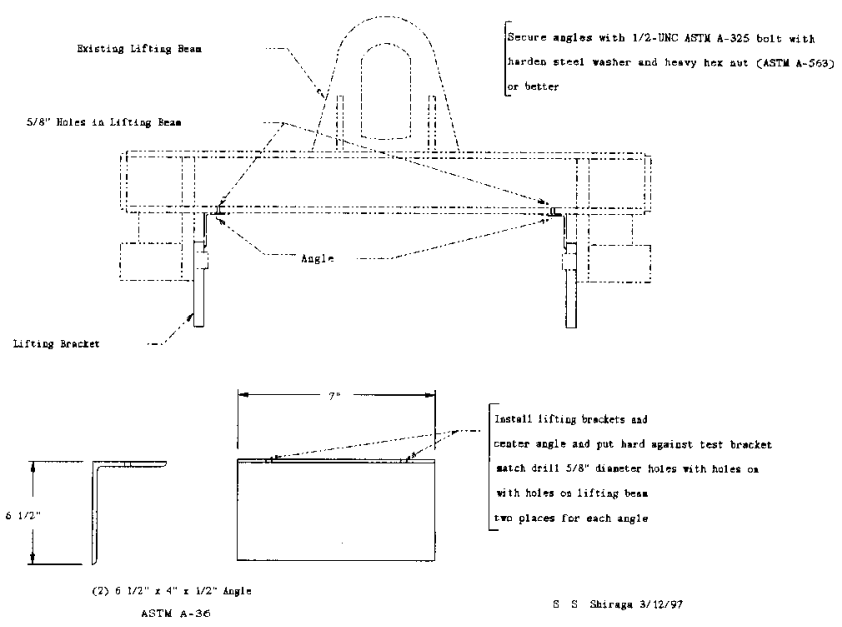

\subsection{REFERENCES}

ANSI, 1993, American National Standard for Radioactive Materials - Special Lifting Devices for Shipping Containers Weighing $10,000 \mathrm{lb}(4500 \mathrm{~kg}$ ) or More, ANSI N14.6, American National Standards Institute, New York, New York.

HNF-SD-TP-SARP-021, Safety Analysis Report for Packaging (Onsite) CASTOR GSF Cask, Rust Federal Services Inc. Northwest Operations, Richland, Washington.

RL, 1993, Hanford Site Hoisting and Rigging Manual, DOE/RL-92-36, U.S. Department of Energy, Richland Operations Office, Richland, Washington. 


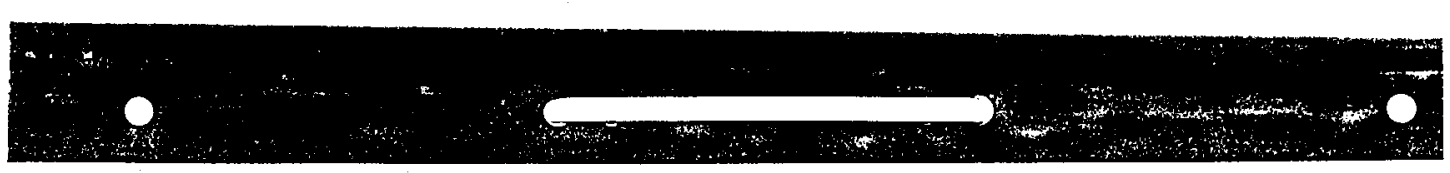

Prüfnáchweis für Lastaúfnahme- und Anschlagmittel gem. §40 vBG 9a

Betriebsmiltei-Nr.

\begin{tabular}{|c|c|c|c|c|c|}
\hline \multirow[b]{2}{*}{ Kette } & \multicolumn{2}{|c|}{$\because$} & \multicolumn{2}{|c|}{ Nr.T } & \multirow[b]{2}{*}{... } \\
\hline & كل & Band $\square$ & Traverse $\mathrm{XI}$ & Gehänge $\square$ & \\
\hline
\end{tabular}
Hersteller/Lieferer Eiscnbau GmbI

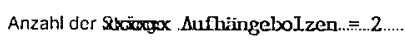

Werksabnahmezeugnis

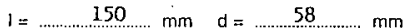

Werkslolf RSt $37-2$

Traglähigkeil deSXgrkxexmepp

Güteklassc

Exxerayaxys

$20.000 \ldots \ldots \ldots \ldots$

Prültast

20.000

Fabr.

דorox-Nr. $\quad 17873$

Gesamitraglähigkeit be

Typ

Baujahr $19 \quad 87$

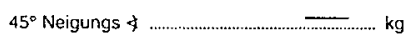

Eigengewicht

ca. 250

$60^{\circ}$ Neigungs $\Varangle$

.................. $\mathrm{kg}$

Inslandsetzung und Wärmebehandlung von vom Fachausschuß Eisen und Melall, SachgeKelten der Güleklasse 3, 5, 6 und 8 nur von biet Kelten, ermächtigt sind.

Kettenherstellern und Werkstätten, die hierzu

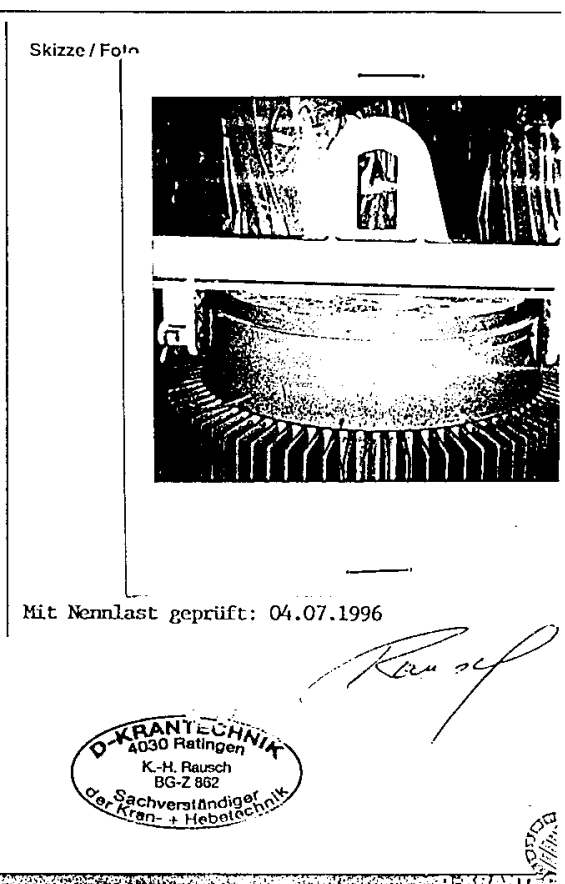

D-KRANTECHNIK D-KRANTECHNIK AG

Osi Schweiz

Am Goethehaus 3

D-99438 Bad Berka

Fox: $(036450) 21153$

Ruchstuckstr.

$\mathrm{CH}-8306$ Brütisellen

Tel.: $(004411) 8333757$

Fax: $(00411)$ \& 330804

Fax: $(036450) 21153$

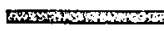

2702 


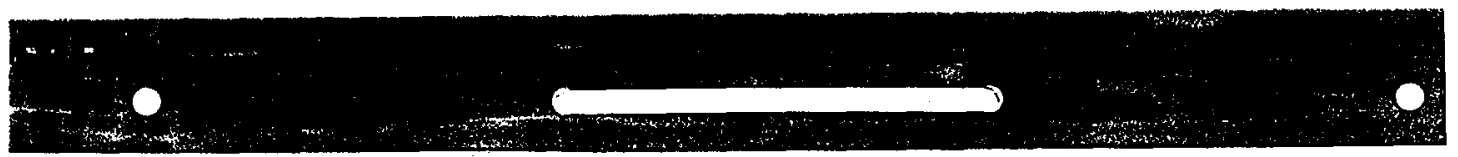

\section{Prüfnachweis für Lastaufnahme- und Anschlagmittel gem. 540 VBG 9a}

Betriebsmittel-Nr. ..............

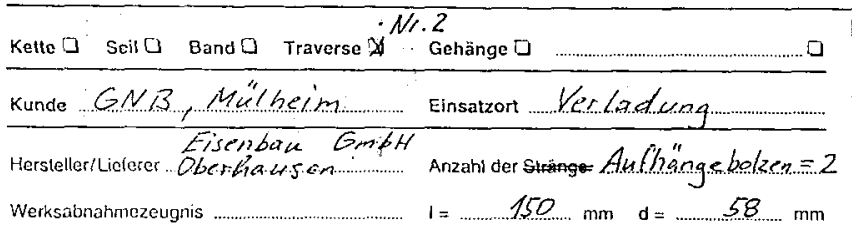

werkstofl $P S t 37-2$ raglähigkeit des

Gütckiasse

Zubehör

Fabr
Zacita.-Nr.

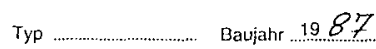

Eigengewicht

ca, 250

Instandsetzung und Wärmebehandlung von Ketten der Gütekiasse 3,5,6 und 8 nur von Kettenherstellern und Werkstätten, die hierzu

vom Fachausschuß Eisen und Melall, Sachgebiet Ketten, ermächtigt sind.

Erate. $20.000 . \mathrm{kg}$ Prüllast $20.000 \mathrm{~kg}$

Gesamıtraglähigkeit bei

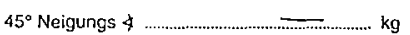

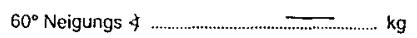
.

Skizze / Foto

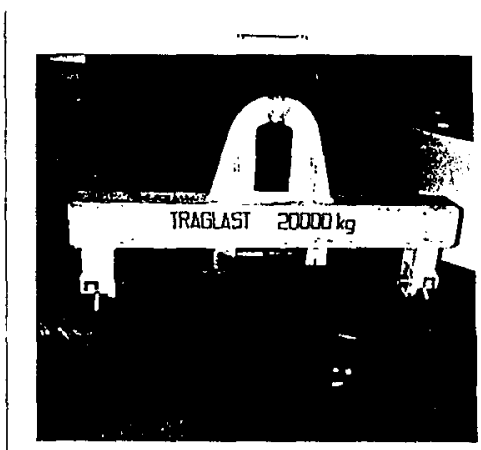

D.kRANTEChNIK Wesl und Nord Setvice-Zentrale Homberger Sitr. 6

D-40B82 Ralingen

Tel.: 102102) 85 $\mathrm{es}+0$

\begin{tabular}{|c|c|c|}
\hline D-KRANTECHKIK & D.KAANTECHNIK & D-KRANTECHNIK AC \\
\hline Sưd & Ost & Sehweiz \\
\hline Theodor-Heuss-Str, 1 & Am Goelhehaus 3 & Ruchstuckstr, 19 \\
\hline D.74139 Schwaigern & D. 99438 Bad Berka & CH.8306 Brühistliten \\
\hline Tet: $(07138) 4011$ & Tol.: $(036458) 31007$ & Tel.: (004 11) B 333757 \\
\hline Fax: $(07138) 4015$ & Fax: $(036458) 31008$ & Fax: $(00411) 6330004$ \\
\hline
\end{tabular}

Mit Nennlast gepinift:

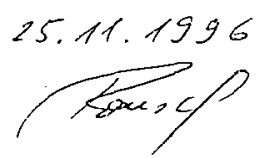

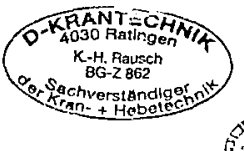

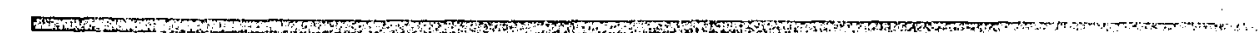




\subsection{HANFORD LOAD TESTS}

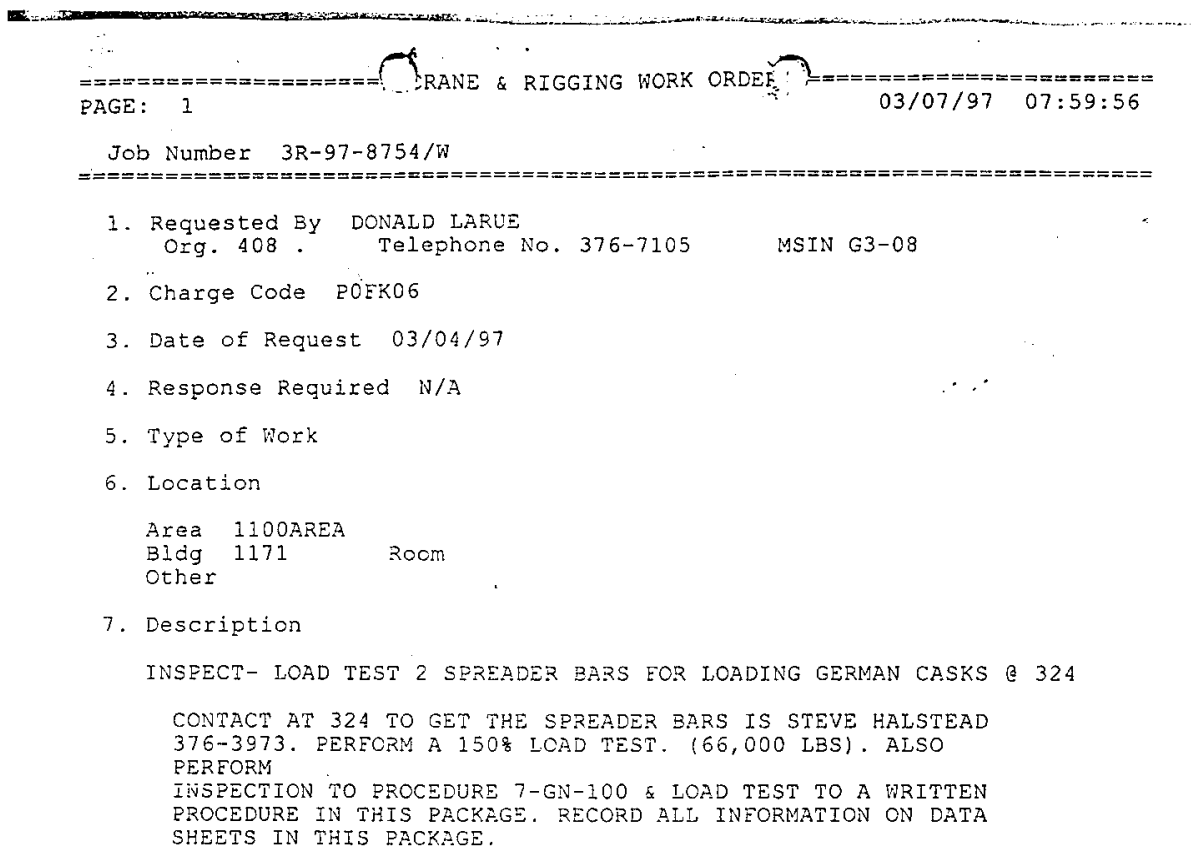
8. Released by
9. Craft Complete
10. Field Work Complete Cancelled
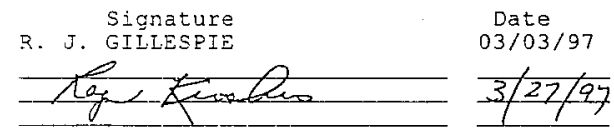

11. Resources Required

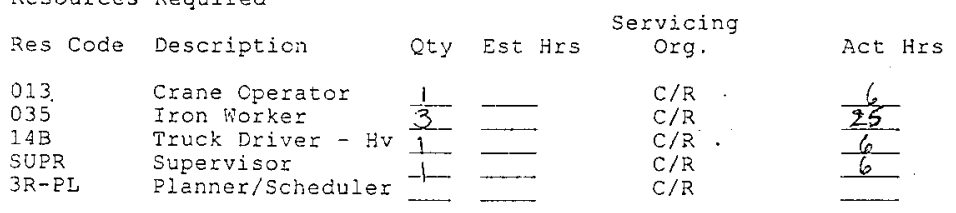




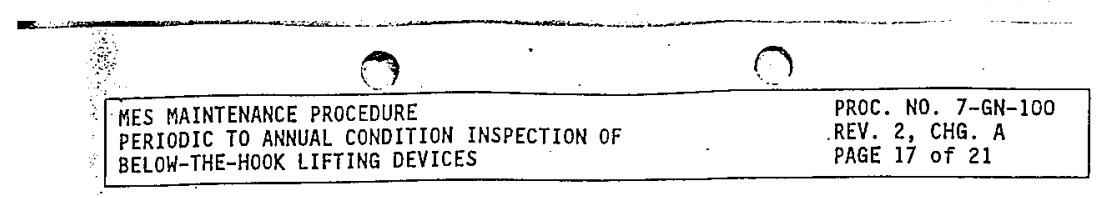

\section{DATA SHEET (Sheet I of 5)}

7.0 INSTRUCTIONS (Record S - Satisfactory, U - Unsatisfactory or N/A - Not Applicabie on steps below).

\begin{tabular}{|c|c|c|c|}
\hline STEP & $S-U-N / A$ & COMMENTS & INITIAL/DATE \\
\hline 7.1 .1 & $S$ & $O K$ & RKIL-3/2ulal \\
\hline 7.1 .2 & $S$ & $O K$ & Rere 3/26/9) \\
\hline 7.1 .3 & $S$ & OK & RKK $3 / 26 / 47$ \\
\hline 7.1 .4 & $S$ & $O K$ & Rke 3/26/97 \\
\hline $7.2 .1 . \mathrm{a}$ & $A / A$ & & \\
\hline$b$ & $n / 4$ & & \\
\hline c & & & \\
\hline 4 & & & \\
\hline $\mathrm{e}$ & & & \\
\hline$f$ & $k / A$ & & \\
\hline$g$ & $n / A$ & & \\
\hline $7.3 .1 . \mathrm{a}$ & $W / A$ & & \\
\hline $\mathrm{b}$ & $n / n$ & & \\
\hline$c$ & $n / A$ & & \\
\hline d & $n / a$ & & \\
\hline e & $n / A$ & & \\
\hline $\mathrm{f}$ & $n / A$ & $\cdot$ & \\
\hline$g$ & $M / A$ & & \\
\hline h & $n / A$ & & \\
\hline 7.3 .2 & $n / A$ & & \\
\hline
\end{tabular}


HNF-SD-TP-RPT-026 Rev. 0

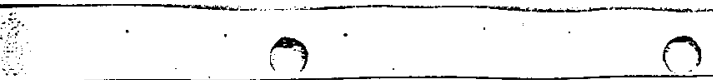

MES MAINTENANCE PROCEDURE

PERIODIC TO ANNUAL CONDITION INSPECTION OF

BELOH-THE-HOOK LIFTING DEVICES

PROC. NO. 7-GN-100

REV. 2, CHG. A

PAGE 18 of 21

DATA SHEET (Sheet 2 of 5)

7.0. INSTRUCTIONS (Record S - Satisfactory, U - Unsatisfactory or N/A - Not Applicable on steps below).

\begin{tabular}{|c|c|c|c|}
\hline STEP & $S-U-N / A$ & COMMENTS & INITIAL/DATE \\
\hline $7.4 .1 . a$ & $n / 14$ & & \\
\hline $\mathrm{b}$ & $n / A$ & & -- \\
\hline c & $/ A$ & & \\
\hline d & & & \\
\hline e & & 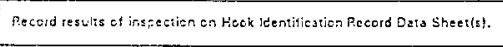 & \\
\hline$f$ & $n / A$ & Recorit resulis of inspection an Haok identificastion Record Dota Sheethat. & \\
\hline 7.4.2.a & $n / 4$ & 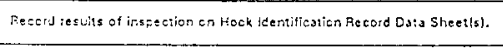 & \\
\hline $\mathrm{b}$ & & 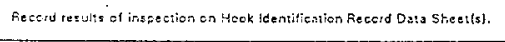 & \\
\hline c & n & 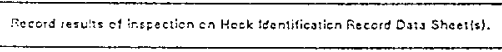 & \\
\hline 7.4 .3 & $n / f$ & 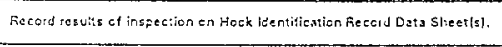 & \\
\hline $7.4 .4 . a$ & $n / n$ & & \\
\hline b & $1 / A$ & & \\
\hline c & $1 / 1$ & & \\
\hline d & & & \\
\hline $7.5 .1 . \mathrm{a}$ & & 05 & RkK 3/2k/92 \\
\hline b & 8 & $O K$ & Rick 3/26/97 \\
\hline c & $n / A$ & . & \\
\hline$d$ & $S$ & Ok & RkK $3 \sqrt[20 / a s]{ }$ \\
\hline e & $n / A$ & & \\
\hline$f$ & $S$ & $0 \leqslant$ & RKK 3/2497 \\
\hline$\underline{g}$ & $S$ & $O K$ & Rkik $3(2 k / \pi)$ \\
\hline
\end{tabular}


HNF-SD-TP-RPT-026 Rev. 0

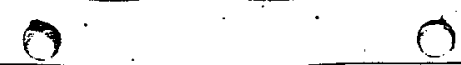

MES MAINTENANCE PROCEDURE

PERIODIC TO ANNUAL CONDITION INSPECTION OF

BELOH-THE-HOOK LIFTING DEVICES

PROC. NO. 7-GN-100

REV. 2, CHG. A

PAGE 19 of 21

\section{DATA SHEET (Sheet 3 of 5)}

7.0 INSTRUCTIONS (Record S - Satisfactory, U - Unsatisfactory or N/A - Not Applicable on steps below).

\begin{tabular}{|c|c|c|c|}
\hline STEP & $S-U-N / A$ & COMMENTS & INITIAL/DATE \\
\hline $7.5 .2 . a$ & 5 & . ok & $R K K-3 / 26 / 47$ \\
\hline$b$ & 5 & $0 K$ & ekic $3 / 2 e / 47$ \\
\hline $\mathrm{c}$ & 5 & $0 K$ & GRK $3 / 2 \mathrm{C} / 97$ \\
\hline d & $\pi / A$ & $\bar{T}$ & \\
\hline e & $n / A$ & & \\
\hline$f$ & $w / k$ & & \\
\hline
\end{tabular}

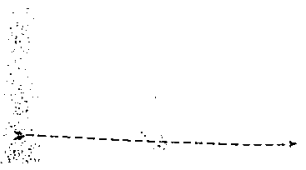

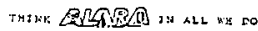




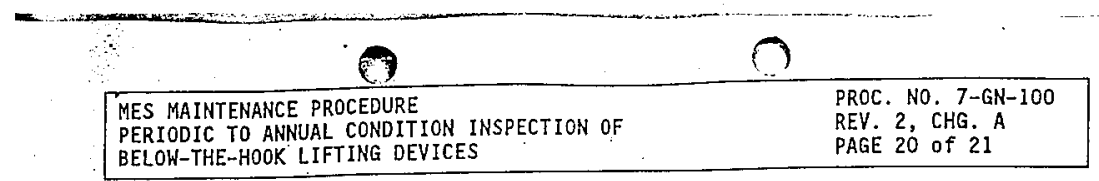

\section{DATA SHEET (Sheet 4 of 5 )}

HOOK IOENTIFICATION RECORD

Inspection Type (Circle one): Initial/Monthly/Periodic (Specify return to service, pre-Critical Lift, etc.)

Inspection Date

Hoist $14 \mathrm{fg}$.

Hoist Serial No.

Hoist Capacity

Hook Dimension "A"

Hook Dimension " $C$ "

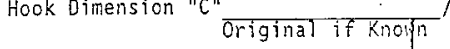

Hook Location/B]dg.

Hook I.D. No.

Hook Capacity

Hook $\mathrm{Mfg}$.

Hook Dimension "B" __

Caliper Serial Wo. Hook NDE Accept Qualified Inspector

NOTE: *Place numbers on hopk as flos th tip of hook as practical.

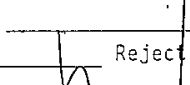
1

1. Sfe attechnent 1 or 2 for disconinuity regair guidet ires.

2. At hock is twisted more then 10 degrees, replace hook.

3. lieasure distance betheen 'B' punchmarks using calipers. The masurement betheen 'A' punchmarks is a reference standerd to compare this dimension with.
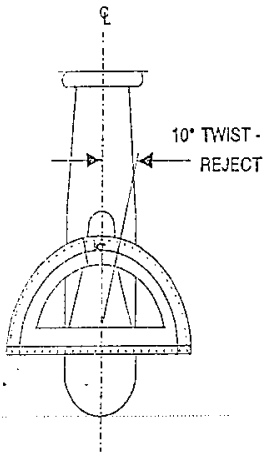
Inspection Type (Circle one): Initial/Monthly/Periodic (Specify return to service, pre-Critical Lift, etc.)

Inspection Date Hoist Mfg. Hoist Serial No. Hoist Capacity Hook Dimension "A" Hook Dimension " $\mathrm{C}$ "

Caliper Serial lio. Hook INE Accept Qualified Inspector NOTE: *Place nunbers on holk as Hook Location/Bidg. Hook I. D. No. Hook Capacity Hook iffg. Hook Dimension "B"

\section{Current}

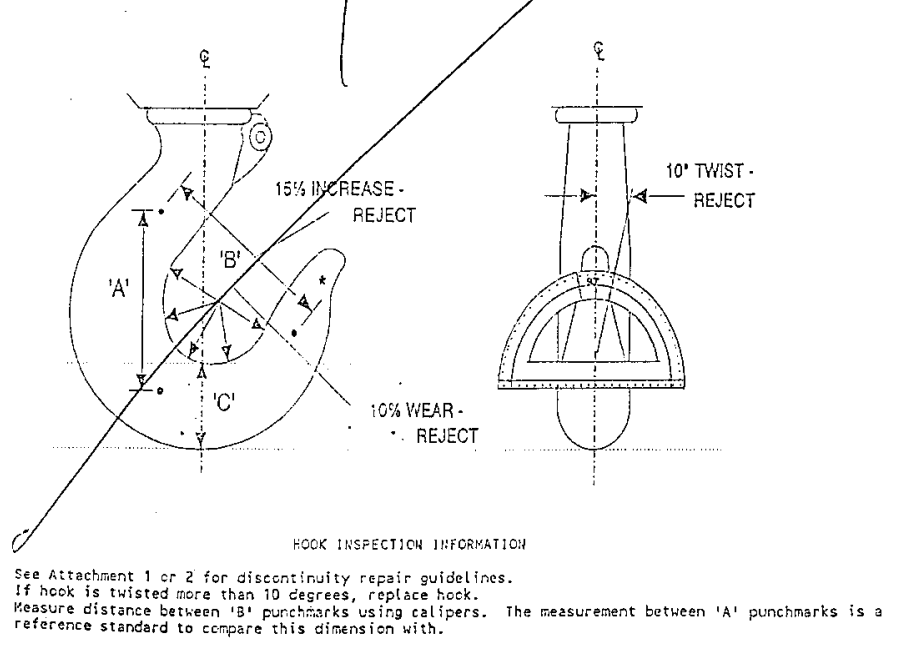

1. See httachment 1 or 2 for discentinuity repair guidelines.

2. If hook is twisted more than 10 decrees, replace took.

reference standard to cempare this dimension with.

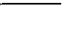


HNF-SD-TP-RPT-026 Rev. 0

A.

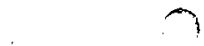

LOAD TEST PROCEDURE

BELOK-THE-HOOK LIFTIKG DEVICE

approval: $\frac{n / A V .6}{\text { Factity tienager }}$

approval: $\frac{M / A}{A G G}$

APPROVAL:

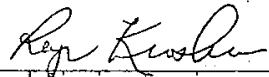

Crane and igging Services

Hanager

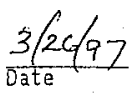



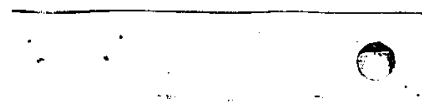

\section{LOED TEST INSTRUCTIONS}

\subsection{PURPOSE}

1.1 The purpose of this procedure is to provide a sequence of operations for load testing a below-the-hook lifting device.

\subsection{REFERENCES}

2.1 Hanford Site Hoisting and Rigging Hanual DOE-RL-92-36

2.2 WHC-CM-4-4, VOL. 1-3, Industrial Safety Manual

2.3 Environmental, Safety, and Health Program lianual.

\subsection{RESPONSIBILITIES}

3.1 The equipment custodian (designated by the facility mancerer) is responsible for ensuring maintenance inspections and testing of equipment are not delinquent. He is also responsible for maintaining records of the repairs, inspections, tests, and any maintenance performed. - He will assure these records are available for audit.

3.2 Industrial Safety shall ensure compliance with hoisting and rigging equipment requirements.

3.3 A designated leader shall be appointed to all hoisting and rigging (H\&R) activities, which include both critical and noncritical lifts. For critical lifts, the designated leader may also be the PIC. For ordinary lifts, the designated leader may be a crely member.

3.4 Designated Leader or (if needed) Site Crane and Rigging Services (SCARS) Supervisor/or Designee is responsible for (1) coordinating the test lift, (2) Ensuring a procedure is prepared and approved, (3) ensuring that personnel are qualified to perform the hork, (4) ensuring that all equipment and rigging are qualified.

\subsubsection{Designated Leader or Supervisor shall conduct a pre-job} meeting.with all personnel involved in the test.

3.5 QC/NDE shall conduct NDE test of nelds after load test. RDE requirements, if required, will be called out on design drawing or listed on the rork package. 


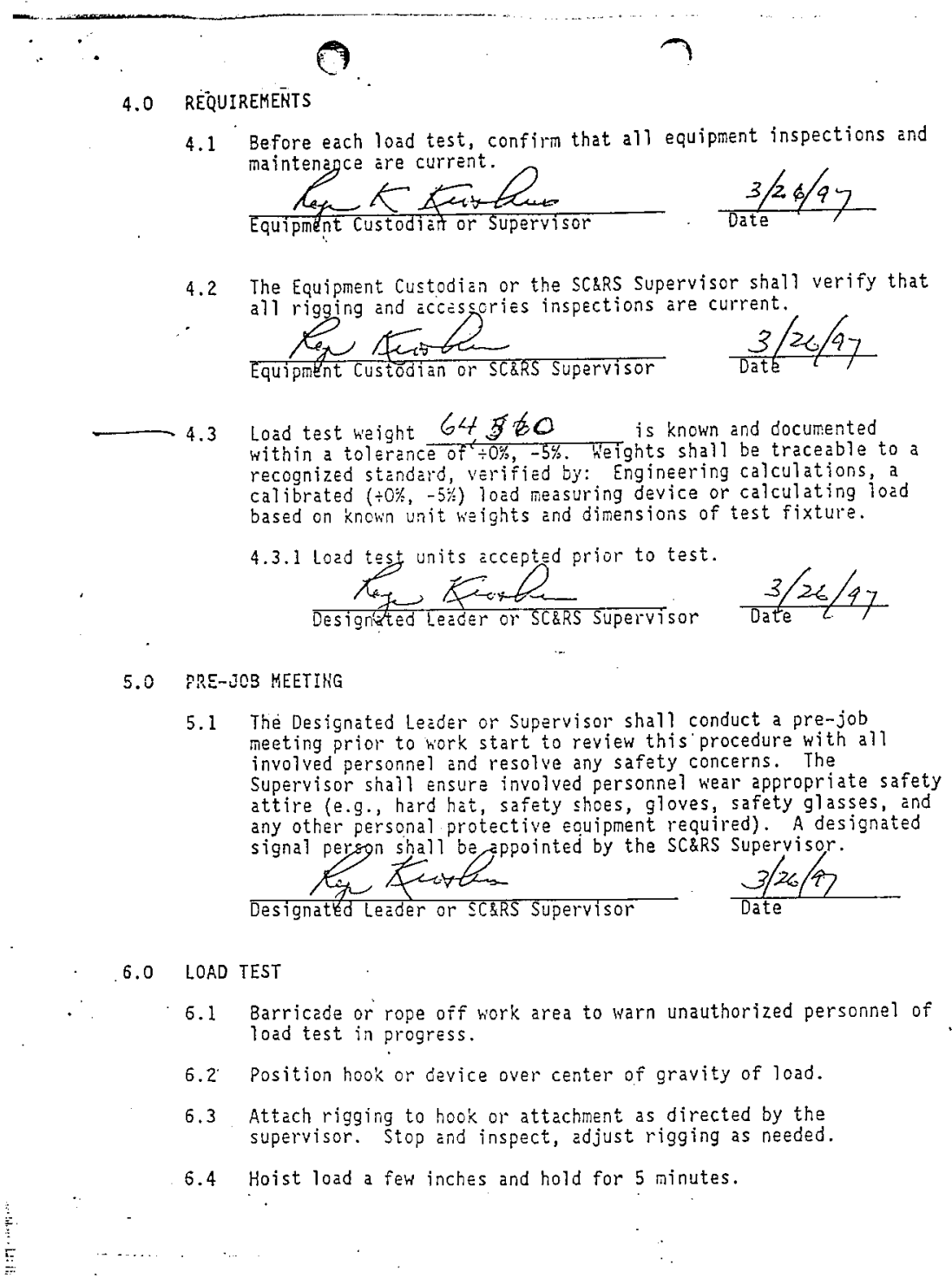




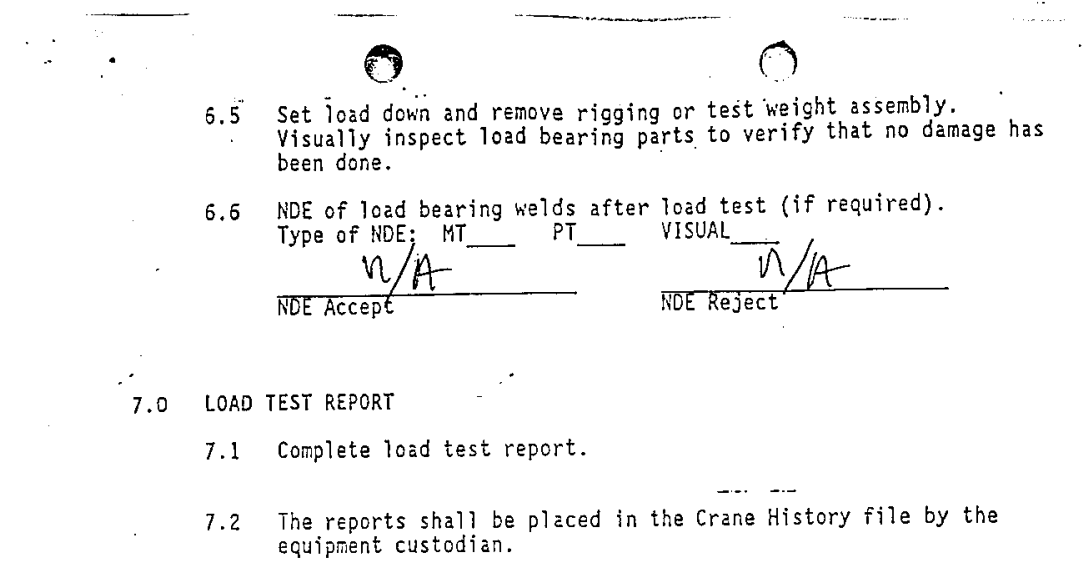

7.1 Complete load test report.

7.2 The reports shall be placed in the crane History file by the
Equipment custodian. 

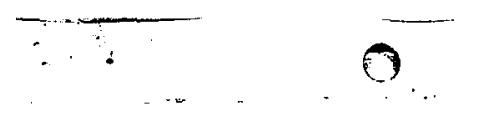

\section{Rev. 0}

\section{LOAD TEST REPORT FORM}

* Building lo. /Facility $324 \mathrm{~B} / \mathrm{dg}$. Load Test Date 3-26-97

*I.O. Ho. 1-A Model No. V/A Manufacturer Hersteller

* Rated Capacity_ 44,000

* Last Load Test Date . n/A Keight(s) Lifted 64,560

* This Load Test: Weight Lifted 64,560

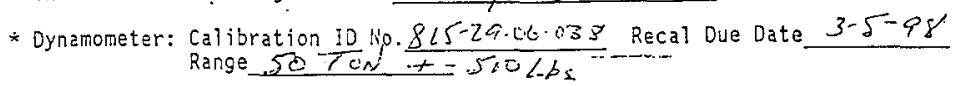
Remarks:

\section{[] Quality Assurance}

[ ] Third Party Verification

(Check one)

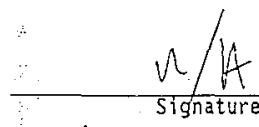

After Load Test Place This Document in the Crane History File. 
HNF-SD-TP-RPT-026 Rev. 0

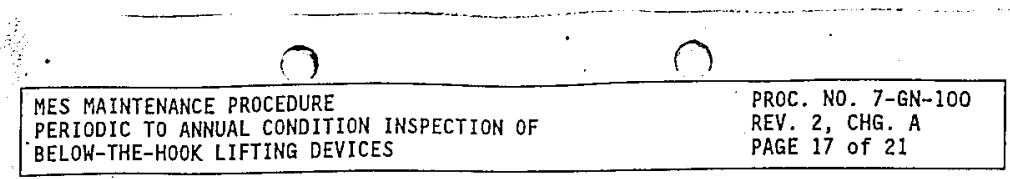

\section{DATA SHEET (Sheet 1 of 5 )}

7.0 INSTRUCTIONS (Record S - Satisfactory, U - Unsatisfactory or N/A - Not Applicable on steps belory).

\begin{tabular}{|c|c|c|c|}
\hline STEP & $S-U-N / A$ & COMMENTS & INITIAL/DATE \\
\hline 7.1 .1 & 5 & $O K$ & $R_{k 1}-3 / 2 k / a_{7}$ \\
\hline 7.1 .2 & $S$ & $0 K$ & RICK $3(26) 97$ \\
\hline 7.1 .3 & $S$ & OK & RKK 3/26/97 \\
\hline 7.1 .4 & 5 & OK & $A_{K K} 3.26 .47$ \\
\hline \multicolumn{4}{|c|}{ 7.2.1.a } \\
\hline$b$ & $n / A$ & & \\
\hline \multicolumn{4}{|c|}{$c$} \\
\hline \multicolumn{4}{|c|}{$d$} \\
\hline \multicolumn{4}{|c|}{$\mathrm{e}$} \\
\hline \multicolumn{4}{|c|}{$f$} \\
\hline \multicolumn{4}{|c|}{$\mathrm{g}$} \\
\hline \multicolumn{4}{|l|}{$7.3 .1 . \mathrm{a}$} \\
\hline \multicolumn{4}{|c|}{$\mathrm{b}$} \\
\hline \multicolumn{4}{|c|}{$c$} \\
\hline \multicolumn{4}{|c|}{$d$} \\
\hline \multicolumn{4}{|c|}{ e } \\
\hline \multicolumn{4}{|c|}{$\angle / A$} \\
\hline \multicolumn{4}{|c|}{$n / A$} \\
\hline \multicolumn{4}{|c|}{$\pi / 1$} \\
\hline 7.3 .2 & $n / A$ & & \\
\hline
\end{tabular}

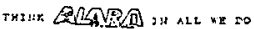


PROC. NO. 7-GN-100

REV. 2, CHG. A

PAGE 18 of 21

\section{DATA SHEET (Sheet 2 of 5 )}

7.0 INSTRUCTIONS (Record S - Satisfactory, U - Unsatisfactory or N/A - Not Applicable on steps below).

\begin{tabular}{|c|c|c|c|}
\hline STEP & $S-U-N / A$ & COMMENTS & INITIAL/DATE \\
\hline \multicolumn{4}{|l|}{$7.4 .1 . \mathrm{a}$} \\
\hline$b$ & $n / A$ & & -- \\
\hline \multicolumn{4}{|c|}{$r$} \\
\hline \multicolumn{4}{|l|}{$d$} \\
\hline e & $n / A$ & Record teeults ef inspection en Hock ldentificestion Fecerd Data Shettiss. & \\
\hline f & $n / A$ & 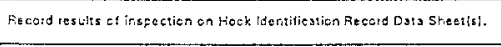 & \\
\hline $7.4 .2 . \mathrm{a}$ & & 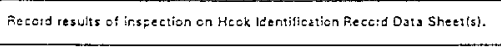 & \\
\hline $\mathrm{b}$ & $n / A$ & 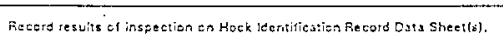 & \\
\hline $\begin{array}{c}c \\
\end{array}$ & & 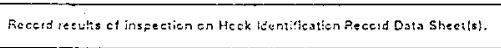 & \\
\hline 7.4 .3 & & 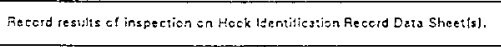 & \\
\hline \multicolumn{4}{|l|}{ 7.4.4.a } \\
\hline \multicolumn{4}{|l|}{ b } \\
\hline \multicolumn{4}{|l|}{ c } \\
\hline \multicolumn{4}{|l|}{ d } \\
\hline $7.5 .1 . a$ & $S$ & $O K$ & Exk 3.264) \\
\hline b & $S$ & OK & RKK 3.26.97 \\
\hline$c$ & $n / A$ & 612 & \\
\hline d & $S$ & OK & RKK $3 \cdot 26-47$ \\
\hline e & $n / A$ & & \\
\hline $\mathrm{f}$ & 5 & $0 k$ & RKK $3.26-97$ \\
\hline $\mathrm{g}$ & 5 & OK & $2 k k 3-26-97$ \\
\hline
\end{tabular}


HNF-SD-TP-RPT-026 Rev. 0

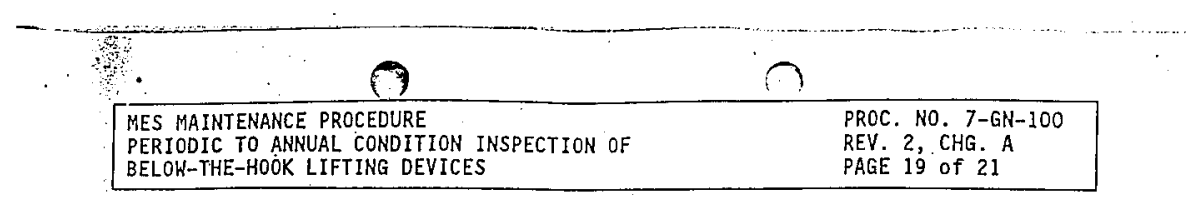

\section{DAIA SHEET (Sheet 3 of 5)}

7.0 INSTRUCTIONS (Record S - Satisfactory, U - Unsatisfactory or N/A - Not Applicable on steps belowv.

\begin{tabular}{|c|c|c|c|}
\hline STEP & $S-U-N / A$ & COMMENTS & INITIAL/DATE \\
\hline $7.5 .2 . a$ & $S$ & $D K$ & $R K K=3 / 22 / 97$ \\
\hline$b$ & $S$ & $O K$ & RKIC 326497 \\
\hline c & $S$ & OK & $R(c k 3 / 20 / 9)$ \\
\hline$d$ & $M / A$ & & \\
\hline $\mathrm{e}$ & $n / 1$ & & \\
\hline$f$ & $n / A$ & & \\
\hline
\end{tabular}




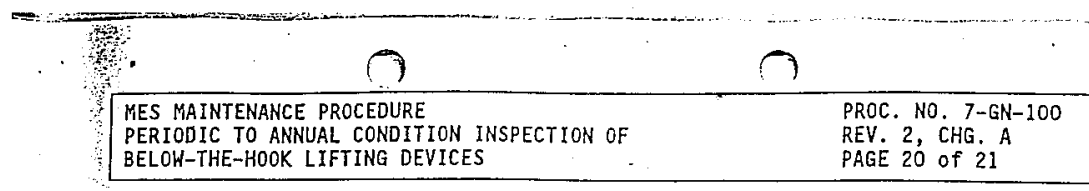

\section{DATA SHEET (Sheet 4 of 5 )}

HOOK IDENTIFICATION RECORD

Inspection Type (Circle one): Initial/Monthly/Periodic

(Specify return to service, pre-[ritical Lift, etc.)

Inspection Date

Hoist $\mathrm{Mfg}$.

Hoist Seria] No.

Hoist Capacity

Hook Dimension "A"

Hook Dimelision "C"

Caliper Serial No. Hook NDE Accept

Qualified Inspector

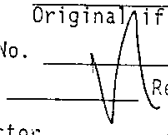

ins on hook

NoTE: xplace numbers on hook as close to tis of hook as practical.

Hook Location/BTdg.

Hook I.D. No.

Hook Capacity

Hook lifg.

Hook Dimension "B"

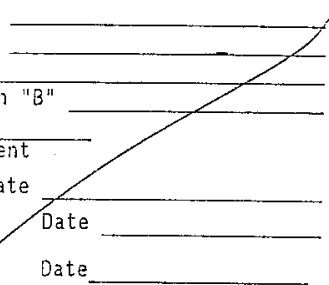

Calibration Date

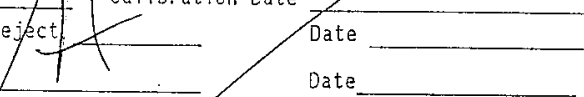

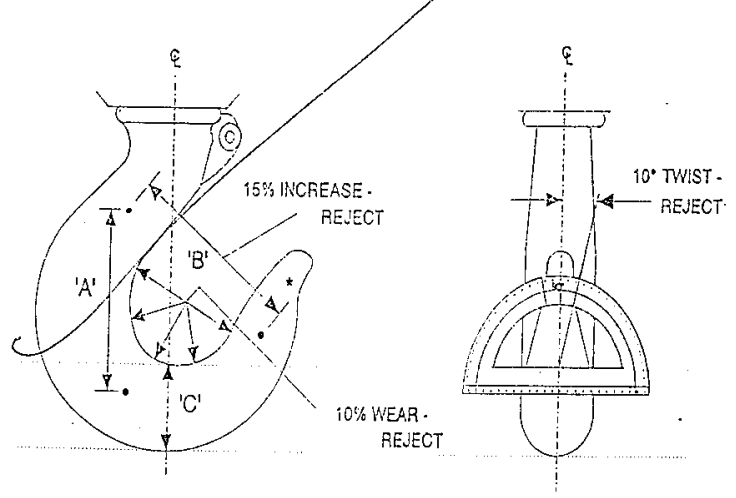

HCOK INSPECTICW IMFOKMATIOH

1. See Attachnent 1 or 2 for discontinuity repoir guidelines.

3. It hook is twisted more than to degrees, replace hook.

reasure distance between 'B' punchwarks using calipers. The inessurement between 'A' punchmorks is a 


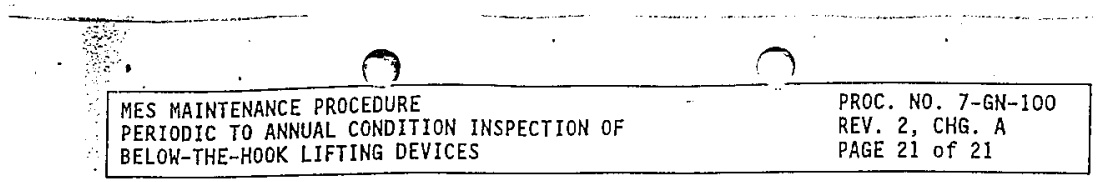

\section{DATA SHEET (Sheet 5 of 5 )}

HOOK IDENTIFICATION RECORD

Inspection Type (Circle one): Initial/Monthly/Periodic (Specify return to service, pre-Critical Lift, etc.)

Inspection Date Hoist Mfg.

Hoist Serial No.

Hoist Capacity

Hook Dimension "A"

Hook Dimension " $\mathrm{C}$ "

Caliper Serial No.

Hook NOE Accept Qualified Inspector

NOTE: *Place numbers on hook as close to tif of hook as practical.

Hook Location/B]dg.

Hook I.D. Wo.

Hook Capacity

Hook Mfg.

Hook Dimension "B"

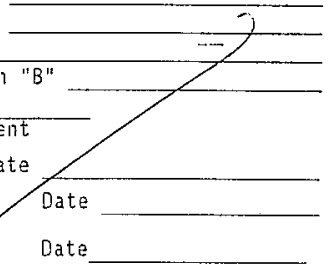

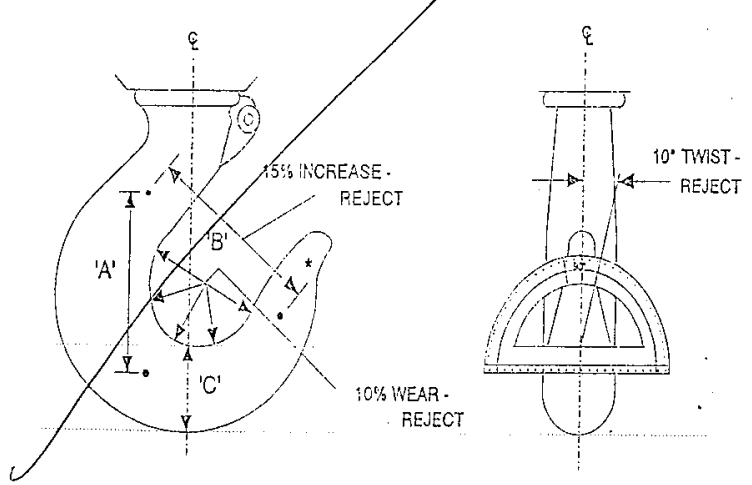

HOCK INSFECTION IHFORHATLOH

1. See fitachment 1 or 2 for discentisuity refair cuidelines.

3. If hook is twisted more than 10 desrees, replsce hook.

Heasure distance betheen 'B' Funchmerks using calipers. The measurement between 'Ad punchmarks is a
reference standard to compere this dimensicn itith.

is

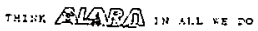

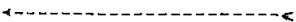


HNF-SD-TP-RPT-026 Rev. 0

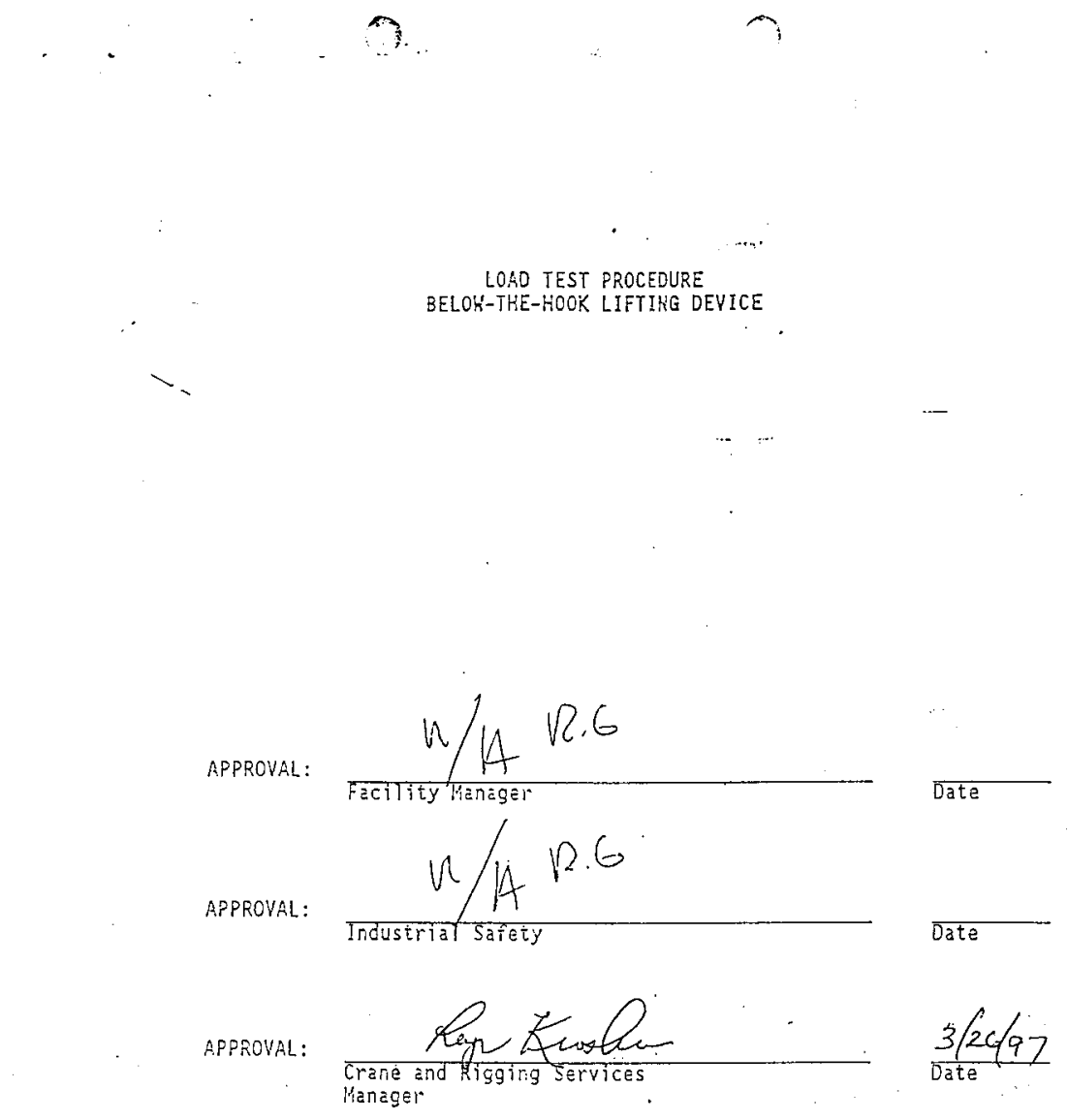


HNF-SD-TP-RPT-026 Rev. 0
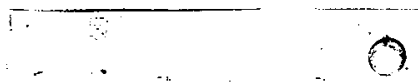

LORD TEST THSTRUCTIONS

\subsection{PURPOSE}

1.1 The purpose of this procedure is to provide a sequence of operations for load testing a below-the-hook lifting device.

\subsection{REFERENCES}

2.1 Hanford Site Hoisting and Rigging Hanual DOE-ŔL-92-36

2.2 HHC-CH-4-4, VOL. 1-3, Industrial Safety Hanual

2.3 Environmental, Safety, and Heath Progran lisnual.

\subsection{RESPOHSIBILITIES}

3.1 The equiprent custodian (designated by the facility manager) is responsible for ensuring mantenarice inspections and testing of equipnent are not delinguent. Hie is also responsible for mantaining records of the repairs, inspections, tests, and any maintenance performed. - He will assure these recoros are available for eudit.

3.2 Industrial Safety shail ensure compliance nith hoisting and rigging equipinent requirenents.

3.3 A designated leader shall be appointed to all hoisting and rigging (H\&R) activities, which include both critical and noncritical iffts. For critical ilits, the designated leader may also be the PIC. For ordinary lifts, the designated leader may be a crew member.

3.4 Designated Leader or (iî needed) Sita Crane and Rigging Services (SC\&RS) Supervisor/or Designee is responsible for (1) coordinating the test lift, (3) ensuring a procedure is prepared and approved, (3) Ensuring that personnel are gualified to perforn the rork, (4) ensuring that all equipment and rigging are qualified.

\subsubsection{Designated Leader or Supervisor shall conduct a pre-job} meeting with all personnel involved in the test.

3.5 QC/NOE shali conófuct WDE test ố welds after load test. HDE requirements, if reçuired, will be called out on desion drating or listed on the nork package. 


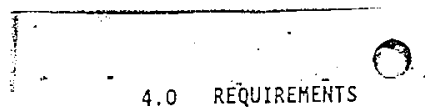

4.1 Before each load test, confirm that all equipment inspections and maintejence are curgent.

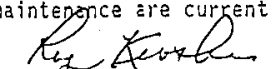

Equipinent Custodian or Supervisor

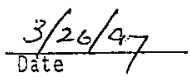

4.2 The Equipment Custodian or the SC\&RS Supervisor shall verify that all rigging end accesscries inspections are currenty.

Equipmedt Custodian or SC SiRS supervisor

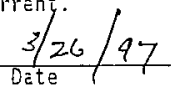

4.3 Load test weight 64560 is known and documented within a tolerance of $\div 0 \%,-5 \%$. Heights shall be traceable to a recognized standard, verified by: Engineering calculations, a calibrated $(\div 0 \%$, $-5 \%)$ load measuring device or calculating load based on known unit weights and dimensions of test fixture:

\subsubsection{Load tast ugits accepted prior to test.

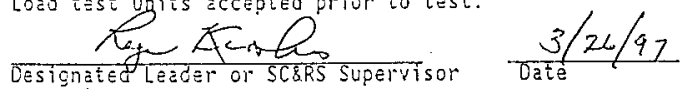

\subsection{PRE-JOB HEETIHE}

5.1 The Desicnated Leader or Superviscr shall conduct a pre-job meeting prior to hork start to reviev this procedure with all involved personnel and resolve any safety concerns. The Supervisor shall ensure involved personnel wear appropriate safety attire (e.g., hard hat, safety shoes, gloves, safety glasses, and any other personal protective eouiprent required). A designated signal pesson shall begippointed by the SC\&RS superyisor.

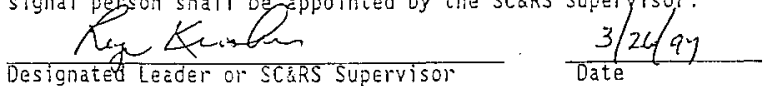

\subsection{LOAD TEST}

6.1 Barricade or rope of trork area to warn unathorized personnel. of load test in progress.

6.2 Position hook or device over center of gravity of load.

6.3. Attach rigging to hook or attachinent as directed by the supervisor. Stop and inspect, adjust rigging as needed.

6.4 Hoist load a feir inches and hold for 5 minutes. 
8

6.5 Set ioad down and remove rigging or test keight assembly. Visually inspect load bearing parts to verify that no damage has been done.

6.6 NOE of loed bearing welds after load test (if required). TYPe of NDE: HTT

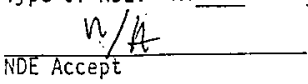
VISUAL

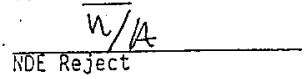

\subsection{LOAD TEST REPORT}

7.1 Complete load test report.

7.2 The reports shail be pleced in the Crane History file by the equipment custodian. 


\section{LOAD TEST REPORT FORM}

* Building No./Facility $324 \mathrm{~B} / \mathrm{dg}$.___L Load Test Date $3-26-97$

* I.D. Ho. 1-B Hodel No. n/A Manufacturer Hersteller

* Rated Capacity 44,000

* Last Load Test Data_M/A._Height(s) Lifted 6 4,560

* This Load Test: Keight Lifted 64,560

* Dynamometer: Calibration ID lio. $815-29-06-038$ Recal Due Date 3-5-98 Renge 50 tent $-\frac{815}{500}$ L135

Remerks:

[ ] Quality Assurance

[ ] Third Party Verification

(Check One)

$\therefore+$

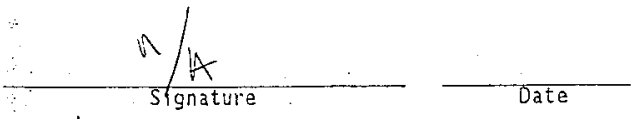

After Load Test Place This Document in the Crane History File. 
HNF-SD-TP-RPT-026 Rev. 0

\subsection{CASTOR LIFTING BEAM EVALUATION}

ENGINEERING SAFETY EVALUATION

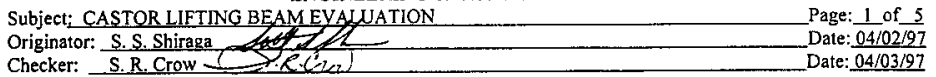

\section{Objective:}

The objective of this evaluation is ensure the CASTOR lifting beam meets the requirements of the Hanford Site Hoisting and Rigging Manual. The requirement is a safety factor of 3 to 1 based on yield strength.

\section{References:}

DOE, DOE-RL-92-36, Honford Site Hoisting and Rigging Manual, Richland Field Office, Richland, WA., January, 1993.

HNF-SD-TP-SARP-021, Safety Analysis Report for Packaging (Onsite) CASTOR GSF Cask, RFS NW Operations, Richland, WA.

Hudson, R. G., The Engineers' Manual, Second Edition, John Wiley and Sons, New York, New York, 1939.

AISC, Manual of Steel Construcrion, Ninth Edition, American Institute of Steel Construction, Chicago, Illinois, 1989.

D-Krantechnik, Lifting Beam Structural Calculations, Ratingen, Germany, May 7, 1996.

III. Results and Conclusions:

Results of this evaluation verifies the CASTOR Lifting Beam meets the requirements of the Hoisting and Rjgging Manual (DOE, 1993). The evaluation is based on the nominal strengths of equivalent German structural steel. As shown in the evaluation the safety factors for the most critical components are equal to or greater than 3. Within this evaluation, the welds are assumed to have the same structural strength as the parent material. Since the welds are not located critical or high load areas, they are not evaluated. 
ENGINEERING SAFETY EVALUATION

SUbject: CASTOR LIFTING BEAM EVALUATION

Page: 2 of 5

Originator: S.S. Shiraga Date:04/02/97

Checker: S.R. Crow 1 te?

Date: $04 / 03 / 97$

IV. Evaluation:

\section{CASTOR LIFTING BEAM EVALUATION:}

Assumed lifting weight of CASTOR w/o impact limiters (CASTOR SARP): $W_{\text {cast }}=41000 \mathrm{lbf}$

Assumed maximum lifting weight of Lifting Beam: $W_{\max }=44000 \mathrm{lbf}$

Beam material (RSt $37-2$ ) yield strength: $\quad s_{37 y}=225 \frac{\text { newton }}{\mathrm{mm}^{2}} \quad s_{37 y}=32.63 \cdot \mathrm{ksi}$
ASTM Equivalent A-570.

Eye plate (St 52-2) yield strength: $\quad s_{52 y}=345 \frac{\text { newton }}{\mathrm{mm}^{2}} \quad s_{52 \mathrm{y}}=50.04 \cdot \mathrm{ksi}$
ASTM Equivalent A-516.

Lifting pin (St 50) yield strength: $\quad$ s $_{50 y}:=275 \frac{\text { newton }}{\mathrm{mm}^{2}} \quad$ s $_{50 \mathrm{y}}=39.89 \cdot \mathrm{ksi}$
ASTM Equivalent A-515.

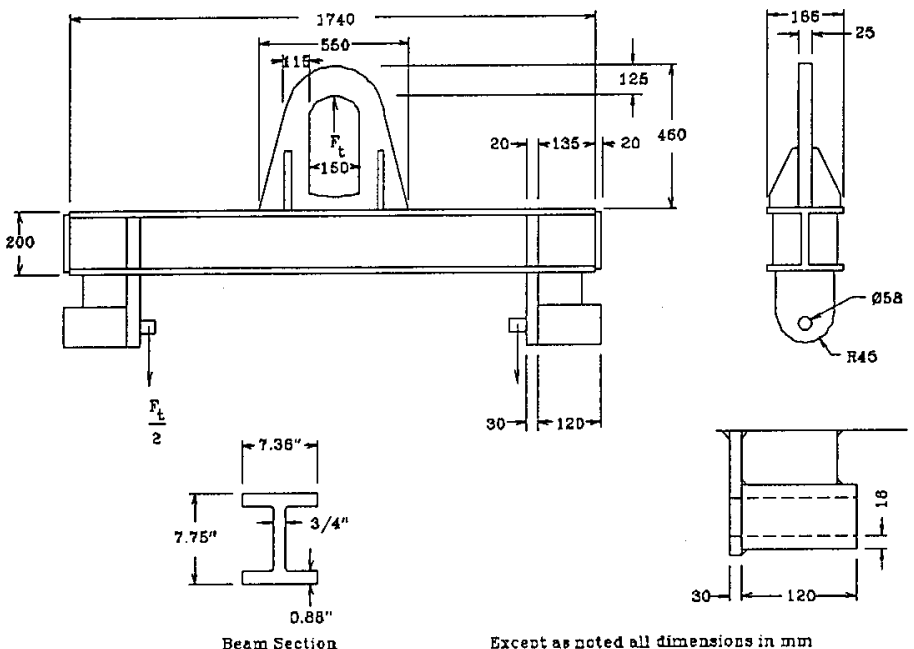




Originator: S.S. Shiraga ___ Date: $04 / 02 / 97$

Checker: S.R. Crow 1 KC:

Determine moment of inertia of $I$ beam section about the axis perpendicular to the web:

Width of flange: $w_{f}=7.38$ in Depth of section: $s_{d}:=7.75$ in $\quad$ Flange thickness: $\quad t_{f}=0.88$ in

Thickness of web: $\quad t_{w}=0.75 \mathrm{in} \quad$ Depth of web: $\quad w_{s}:=s_{d^{-2}}-t_{f}$

Moment of inertia (Hudson, 1939, page 84): $\quad I_{b s}=\frac{w_{f^{s}} d^{3}-w_{s}{ }^{3} \cdot\left(w_{f}-t_{w}\right)}{12} \quad I_{b s}=168 \cdot$ in $^{4}$

Distance of center of gravity: $\quad d_{i c g}:=\frac{s_{d}}{2} \quad d_{i c g}=3.87 \cdot \mathrm{in} \quad$ Section Modulus: $\quad s_{b s}=\frac{I_{b s}}{d_{i c g}}$.

Cross sectional area: $\quad A_{b s}=2 \cdot t_{f} w_{f}+t_{w} \cdot w_{s} \quad A_{b s}=17.48 \cdot i^{2}$

Area of compression flange: $\quad A_{f}=t_{f} w_{f} \quad A_{f}=6.49 \cdot i^{2}$

Evaluate to AISC Design Requirements:

Width to thickness ratio: $\quad \frac{w_{\mathrm{f}}}{2 \cdot \mathrm{t}_{\mathrm{f}}}=4.19 \quad$ Limiting width to thickness ratio: $\quad \frac{65}{\sqrt{\frac{\mathrm{s} 37 \mathrm{y}}{\mathrm{ksi}}}}=11.38$

Since width to thickness ratio not limiting, section is compact.

Determine value of limiting laterally unsupported length for compact section in strong axis bending:

$$
\frac{76 w_{f}}{\sqrt{\frac{s}{37 y}}}=8.18 \mathrm{ft} \quad \text { or } \quad \frac{20000}{\mathrm{~s}_{\mathrm{d} i}} \cdot \frac{\mathrm{s} 37 \mathrm{y}}{\mathrm{Assi}}=42.8 \mathrm{ft}
$$

Unsupported length of I beam: $\quad \mathrm{L}_{b}=1740 \mathrm{~mm} \quad \mathrm{~L}_{b}=5.71 \cdot \mathrm{ft}$

Therefore, since critical length not exceeded flexture allowable of beam is: $s_{37 b}:=0.66 \mathrm{~s} 37 \mathrm{y} \quad \mathrm{s}_{37 \mathrm{~b}}=21.54 \cdot \mathrm{ksi}$

Loading on Main Beam:

Idealize loading as a simply supported beam with a partially distributed uniform load over center section. AISC, 1989, page 2.297.

Length between load: $\quad 1_{1}:=1740 \mathrm{~mm}-2 \cdot(175 \mathrm{~mm}) \quad$ Load width: $\quad b_{1}=550 \mathrm{~mm}$

Distance from load: $\quad a_{1}=\frac{l_{1}-b_{1}}{2} \quad a_{1}=420 \cdot \mathrm{mm}$

Load on unit: $\quad F_{t}=W_{\max } \quad$ Load per unit length: $\quad f_{1}=\frac{F_{t}}{b_{1}} \quad f_{l}=2032 \cdot \frac{\text { lbf }}{\text { in }}$ 


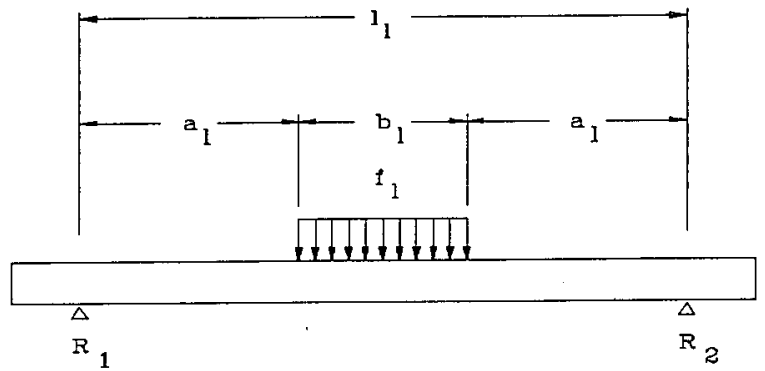

Since symmetrical, reaction load at load points: $\quad R_{1}:=\frac{f_{1} \cdot b_{1}}{2 \cdot l_{1}} \cdot\left(2 \cdot a_{1}+b_{1}\right) \quad R_{1}=22000 \cdot \mathrm{lbf}$

Maximum Moment: $M_{\max }=R_{1} \cdot\left(a_{I} \div \frac{R_{1}}{2 \cdot f_{1}}\right) \quad M_{\max }=482874-\mathrm{lbf} \cdot$ in

Bending stress: $\quad \sigma_{b}=\frac{M_{\max }}{S_{b s}} \quad \sigma_{b}=11.17 \cdot \mathrm{ksi} \quad$ Shear at Edge: $\quad \tau_{b}:=\frac{R_{1}}{A_{b s}} \quad \tau_{b}=1.26 \cdot k s i$

Safety Factor based on Yield Strength: $\quad \mathrm{SF}_{\mathrm{b}}=\frac{\mathrm{s}_{37 y}}{\sigma_{\mathrm{b}}} \quad \mathrm{SF}_{\mathrm{b}}=3$

Loading on Lift Pin:

Assume as cantilevered circular beam that is loaded between cask lifting bearn. Treat as short beam.

Pin diameter: $d_{\text {pin }}:=58-\mathrm{mm} \quad$ Load on pins: $\quad F_{p}:=\frac{F_{t}}{2} \quad F_{p}=22000-1 b f$

Cross sectional area of pin: $\quad A_{p}=\pi \cdot \frac{d p^{2}{ }^{2}}{4} \quad$ Moment of inertia of cross section: $\quad I_{p}=\frac{\pi \cdot d_{\text {pin }}{ }^{4}}{64}$

Distance between pin supports: $\quad 1_{\text {ps }}=1740 \mathrm{~mm}-2 \cdot(20+135+20) \cdot \mathrm{mm}$

Gap between cask and lifting beam: $\quad \mathrm{I}_{\mathrm{g}}=\frac{\mathrm{l}_{\mathrm{ps}}-1365 \mathrm{~mm}}{2} \quad \mathrm{l}_{\mathrm{g}}=0.49 *$ in 
Shear stress on pin: $\quad \tau_{p}=\frac{F_{p} \cdot\left(\frac{d p i n}{2}\right)^{2}}{3 \cdot 1_{p}} \quad \tau_{p}=7.16 . \mathrm{ksi}$

Bending: $\quad \sigma_{p}=\frac{F_{p} \cdot g}{I_{p}} \cdot \frac{d_{p i n}}{2} \quad \sigma_{p}=9.26 * \mathrm{ksi}$

Principal stress: $\quad \sigma_{1}=\frac{\sigma_{p}}{2}+\sqrt{\left(\frac{\sigma_{p}}{2}\right)^{2}+\tau_{p}{ }^{2}} \quad \sigma_{1}=13.2 \cdot \mathrm{ksi}$

Safety Factor based on Yield Strength: $\quad \mathrm{SF}_{\mathrm{p}}=\frac{{ }^{s} 50 \mathrm{y}}{\sigma_{1}} \quad \mathrm{SF}_{\mathrm{p}}=3$

Loading on lifting eye:

Thickness of plate: $\quad t_{e}=25 \mathrm{~mm} \quad$ Length on each side of opening: $\quad I_{e}=115 \mathrm{~mm}$

Distance to outside edge: $\quad d_{\text {oe }}=125 \mathrm{~mm}$

Tensile stess on lifting eye: $\quad \sigma_{\text {et }}=\frac{F_{t}}{2 \cdot t_{\mathrm{e}} \cdot \mathrm{I}_{\mathrm{e}}} \quad \sigma_{\mathrm{et}}=4.94 \cdot \mathrm{ksi}$

Safety Factor based on Yield Strength: $\quad \mathrm{SF}_{\text {et }}:=\frac{s 52 \mathrm{y}}{\sigma_{\text {et }}} \quad \mathrm{SF}_{\text {et }}=10.1$

Shear tearout: $\quad \tau_{\text {tet }}=\frac{F_{t}}{2 \cdot \mathrm{t}_{\mathrm{e}} \cdot \mathrm{d}_{\mathrm{oe}}} \quad \tau_{\text {tet }}=4.54{ }^{*} \mathrm{ksi}$

Safety Factor based on Yield Strength: $\quad \mathrm{SF}_{\text {tet }}=\frac{\mathrm{S}_{52 \mathrm{y}}}{\tau_{\text {tet }}} \quad \mathrm{SF}$ tet $=11$ 
DISTRIBUTION SHEET

\begin{tabular}{|c|c|c|c|c|c|}
\hline \multirow{2}{*}{$\begin{array}{l}\text { To } \\
\text { Distribution }\end{array}$} & \multirow{2}{*}{\multicolumn{3}{|c|}{$\begin{array}{l}\text { From } \\
\text { Packaging Engineering }\end{array}$}} & \multirow{2}{*}{\multicolumn{2}{|c|}{$\begin{array}{l}\text { Page } 1 \text { of } 1 \\
\text { Date } 04 / 04 / 97\end{array}$}} \\
\hline & & & & & \\
\hline \multicolumn{4}{|l|}{ Project Title/Work Order } & \multicolumn{2}{|c|}{ EDT No. 619562} \\
\hline \multicolumn{4}{|c|}{$\begin{array}{l}\text { Spreader Beam Analysis for the CASTOR GSF Cask } \\
\text { (HNF-SD-TP-RPT-026) }\end{array}$} & \multicolumn{2}{|c|}{ ECN No. N/A } \\
\hline Name & MSIN & $\begin{array}{c}\text { Text } \\
\text { With All } \\
\text { Attach. }\end{array}$ & Text Only & $\begin{array}{l}\text { Attach./ } \\
\text { Appendix } \\
\text { Only }\end{array}$ & $\begin{array}{l}\text { EDT/ECN } \\
\text { Only }\end{array}$ \\
\hline $\begin{array}{l}\text { C. E. Brewer } \\
\text { E. P. Clements } \\
\text { J. G. Field } \\
\text { S. D. Halstead } \\
\text { R. K. Kroshus } \\
\text { D. M. LaRue } \\
\text { I. L. Metcalf } \\
\text { S. S. Shiraga } \\
\text { P. J. Weaver } \\
\text { Central Files } \\
\text { HNF-SD-TP-RPT-026 File }\end{array}$ & $\begin{array}{l}\text { S3-15 } \\
\text { H1-15 } \\
\text { HI-15 } \\
\text { L1-02 } \\
\text { G4-07 } \\
\text { G3-08 } \\
\text { L6-26 } \\
\text { H1-15 } \\
\text { L1-02 } \\
\text { A3-88 } \\
\text { H1-15 }\end{array}$ & $\begin{array}{l}x \\
x \\
x \\
x \\
x \\
x \\
x \\
x \\
x \\
x \\
x\end{array}$ & & & \\
\hline
\end{tabular}

